\title{
El examen oftalmológico de rutina no sería preciso para identificar pacientes en riesgo de padecer glaucoma primario de ángulo abierto
}

Routine ophthalmic examination would not be accurate for identifying patients at risk of primary openangle glaucoma

Hollands $\mathrm{H}$ et al. JAMA 2013;309:2035-42.

\section{Objetivo}

Determinar los factores de riesgo para glaucoma primario de ángulo abierto (GPAA) que podrían evaluarse durante un examen clínico de rutina, y la precisión de los hallazgos comunes que podrían ser detectados por el médico general.

\section{Fuentes de datos}

Artículos en inglés de OVID Medline desde enero de 1950 a enero de 2013 que reportaran acerca del valor diagnóstico de hallazgos funduscópicos y puntos de corte de presión intraocular (PIO) para identificar pacientes con glaucoma. Se excluyeron reportes de casos, comentarios, cartas, editoriales y revisiones.

\section{Selección de estudios}

A cada estudio se le asignó un puntaje de calidad. El análisis se realizó sólo sobre estudios de calidad metodológica de nivel 1 y 2: comparación independiente de síntomas o signos con un criterio estándar para glaucoma en un número pequeño y grande: menor o mayor a 1.000, respectivamente de pacientes consecutivos en una población definida.

\section{Extracción de datos}

Se definió "glaucoma" a la presencia de un disco óptico con cambios característicos no explicables por otra causa, asociados a una anomalía correspondiente en el campo visual. En todos los estudios el diagnóstico fue realizado por oftalmólogos, utilizando una evaluación del nervio óptico, campos visuales formales, y medición de la PIO con tonometría de Goldmann (gold standard). Se incluyó también el valor diagnóstico de factores de riesgo para glaucoma (sexo, raza, miopía, antecedentes familiares y comorbilidades sistémicas). Los hallazgos primarios del examen fueron la relación copa-disco (pro- porción que ocupa la excavación papilar en el total de la papila), asimetría en la relación copa-disco, presencia de hemorragia(s) en el disco, y PIO.

\section{Resultados principales}

Se analizaron 50 artículos correspondientes a 34 estudios. No se encontraron estudios de exámenes de rastreo realizados por médicos generales. Los resultados principales se resumen en las tablas 1 a 3.

Tabla 1: prevalencia del glaucoma en los estudios incluidos.

\begin{tabular}{c|c} 
Entidad & Prevalencia \% (IC 95\%) \\
GPAA & $2,6(2,1$ a 3,1$)$ \\
\hline GPAA con PIO $>21 \mathrm{mmHg}$ & $2,4(1,9$ a 2,9$)$ \\
\hline GPAA con PI0 $<21 \mathrm{mmHg}$ & $1,6(1,2$ a 2,1$)$ \\
\hline
\end{tabular}

GPAA: glaucoma primario de ángulo abierto. NR: no reportado.

Tabla 2: factores de riesgo asociados a incremento de riesgo de glaucoma primario de ángulo abierto.

\begin{tabular}{c|c|c} 
Factor de riesgo & Prevalencia \% (IC 95\%) & OR (IC 95\%) \\
\hline Miopía alta (> 6 dioptrías) & $11(8,3$ a 18) & $5,7(3,1$ a 11$)$ \\
\hline Edad avanzada $(>80$ años) & $7,8(5,2$ a 12) & $2,9(1,9$ a 4,3$)$ \\
\hline Antecedentes familiares +a & $7,6(5,3$ a 11$)$ & $3,3(2,0$ a 5,6$)$ \\
\hline Raza negra & $7,5(6,8$ a 8,4$)$ & $2,9(1,4$ a 5,9$)$ \\
\hline
\end{tabular}

OR: Odds Ratio. NR: no reportado. a: ej. familiares de primer grado. Otros factores de riesgo como diabetes mellitus, enfermedad vascular periférica, hábito de fumar, hipertensión y migrañas fueron menos útiles (OR de menor magnitud, o intervalos de confianza que incluían 1,0).

Tabla 3: hallazgos del examen físico y su asociación a glaucoma primario de ángulo abierto.

\begin{tabular}{l|c|c|c}
\multicolumn{1}{c|}{ Hallazgo } & LR + (IC 95\%) & LR - (IC 95\%) & P \\
\hline Aumento de la relación copa-disco $(\geq \mathbf{0 , 7})$ & $14(5,3$ a 39) & 0,62 (0,53 a 0,71) & $<0,01$ \\
\hline Asimetría copa-disco $(\geq \mathbf{0 , 3})$ & $7,3(3,3$ a 16) & $0,89(0,83$ a 0,94) & $<0,001$ \\
\hline Presencia de hemorragia en el disco & $12(2,9$ a 48) & $0,94(0,83$ a 0,98) & NR \\
\hline Presión intra-ocular $\geq 22$ mmHg & $13(8,2$ a 17) & $0,65(0,55$ a 0,76) & NR \\
\hline
\end{tabular}

LR +: likelihood ratio positivo. LR -: likelihood ratio negativo. NR: no reportado.

\section{Limitaciones}

Entre las principales limitaciones: No se encontró evidencia de alta calidad que permitiera determinar la precisión del examen funduscópico realizado por médicos generales; Los estudios utilizados fueron publicados en un período de 40 años, durante los cuales ha mejorado el conocimiento del proceso de la enfermedad y su diagnóstico; Se observó heterogeneidad significativa entre los estudios; Sólo se investigaron estudios diagnósticos aislados como predictivos de GPAA, en vez de combinaciones de exámenes.

\section{Conclusiones}

La presencia de hallazgos individuales como el incremento de la relación copa-disco y su asimetría, las hemorragias en el disco, y la elevación de la PIO, así como la presencia de factores de riesgo demográficos como el antecedente familiar de glaucoma, raza negra y edad avanzada se asocian con un incremento del riesgo de GPAA, pero su ausencia efectiva no lo descarta. La mejor evidencia disponible apoya la evaluación realizada por un oftalmólogo como el método más preciso para detectar glaucoma.

Fuentes de financiamiento: no reportada. Conflictos de interés de los autores: algunos de los autores reportaron recibir apoyo económico por parte de JAMA evidence, Allergan, Alcon y Pfizer. 


\section{Comentario}

En una reciente publicación nos hemos referido ya a características epidemiológicas y clínicas sobre esta entidad'1 Vale recordar que el rastreo de GPAA sería entonces más útil y costo-efectivo si se dirigiera a poblaciones de pacientes con mayor riesgo de desarrollar neuropatía óptica glaucomatosa ${ }^{2}$, tal como sugieren los autores: edad adulta (mayores de 80 años), antecedentes familiares de glaucoma, raza negra, ancestros africanos o etnicidad latina/hispánica, transtornos de la presión arterial, diabetes tipo 2 y miopía alta

\section{Conclusiones del comentador}

La evidencia actual no avala una recomendación de rastreo masivo del GPAA en ámbitos de atención primaria. El rol del médico general en la investigación de estos factores de riesgo es fundamental para aumentar la detección y derivación oportunas al especialista, quien realizará luego el examen completo3. La frecuencia recomendada de la evaluación en este grupo de riesgo es cada 1 a 3 años en personas de 40 a 54 años de edad, cada 1 a 2 años entre los 55 y 64 años de edad, y cada 6 a 12 meses en los mayores de 65 años $^{3}$.

Ana Gabriela Palis [ Servicio de Oftalmología del Hospital Italiano de Buenos Aires. gabriela.palis@ hospitalitaliano.org.ar]

Palis Ana Gabriela. El examen oftalmológico de rutina no sería preciso para identificar pacientes en riesgo de padecer glaucoma primario de ángulo abierto cuando es realizado por médicos generales. Evid Act Pract Ambul. 2015 ;18(1)14-15.Ene-Mar. Comentado de: Hollands H, et al. Do findings on routine examination identify patients at risk for primary open-angle glaucoma? The rational clinical examination systematic review. JAMA. 2013;309(19):2035-42. PMID: 23677315

\section{Referencias}

1. Palis A. Recomendaciones para el rastreo del glaucoma primario de ángulo abierto. Evid Act Pract Ambul. Ene-Mar 2014;17(1)34-36. Comentado de: Moyer V and U.S. Preventive Services Task Force; Screening for Glaucoma: U.S. Preventive Services Task Force Recommendation Statement. Annals of Internal Medicine. 2013 Oct;159(7):484489. PMID: 23836133.

2. American Academy of Ophthalmology Glaucoma Panel. Preferred Practice Pattern® Guidelines. Primary Open-Angle Glaucoma. San Francisco, CA: American Academy of Ophthalmology; 2010. Available at: www.aao.org/ppp.

3. American Academy of Ophthalmology Glaucoma Panel. Preferred Practice Pattern® Guidelines. Primary Open-Angle Glaucoma Suspect. San Francisco, CA: American Academy of Ophthalmology; 2010. Disponible en: www.aao.org/ppp. 